\title{
Do You Know Your New Molecular Entity (NME)?
}

\author{
François Xavier Lacasse $^{1 *}$ and France Guay ${ }^{2}$ \\ ${ }^{1}$ Faculty of Pharmacy, University of Montreal, Canada \\ ${ }^{2}$ President, FG Pharma Inc, Canada
}

Received: 阱 January 10, 2018; Published: 眥 January 24, 2018

*Corresponding author: François Xavier Lacasse, Faculty of Pharmacy, University of Montreal, Canada

\section{Introduction}

During the last decades, life science startup companies have relatively rapidly increased their presence in the pharmaceutical landscape. Pharmaceutical development has evolved because technology (analytical and process development) and clinical development (hybrid design in phase 1, including patients in phase 1B) have evolved in parallel allowing in certain cases to decrease the time to regulatory filings (IND, CTA, NDA, NDA 505 (b)(2),..), which seemed attractive for startup companies. However, new molecular entities have become more complex since polypeptides, proteins, and monoclonal antibodies drug products take more and more place in many therapeutic areas (more than $50 \%$ in the 50 best seller drugs) along with the more conventional small molecules. But it is not the end of small molecules; research institutes, startup companies are still busy working hard to develop more powerful small molecules, since the physiopathology has also evolved and helped finding some new targets to enhance their efficacy and decrease their lack of selectivity. Startup companies are mostly driven by high level scientists and few of them are familiar with the drug development process, including its early phases. Scientists do know their NMEs from a scientific point of view however, much less and, sometimes, not at all in terms of potential drug product candidates.

The authors of this paper cumulate more than 50 years in drug development at various phases with different kind of molecules; they will try to illustrate how what you do not know about your NMEs can and will hurt you and also present how to avoid surprises that may occur down the road of drug development.

\section{People do not know their NME.... however the NME is the most important ingredient of a drug}

These three (3) main questions should be asked:
a) Why do we not know our NME?
b) When and how does it hurt?
c) What can we do to prevent it?
a) Why do we not know our NME?

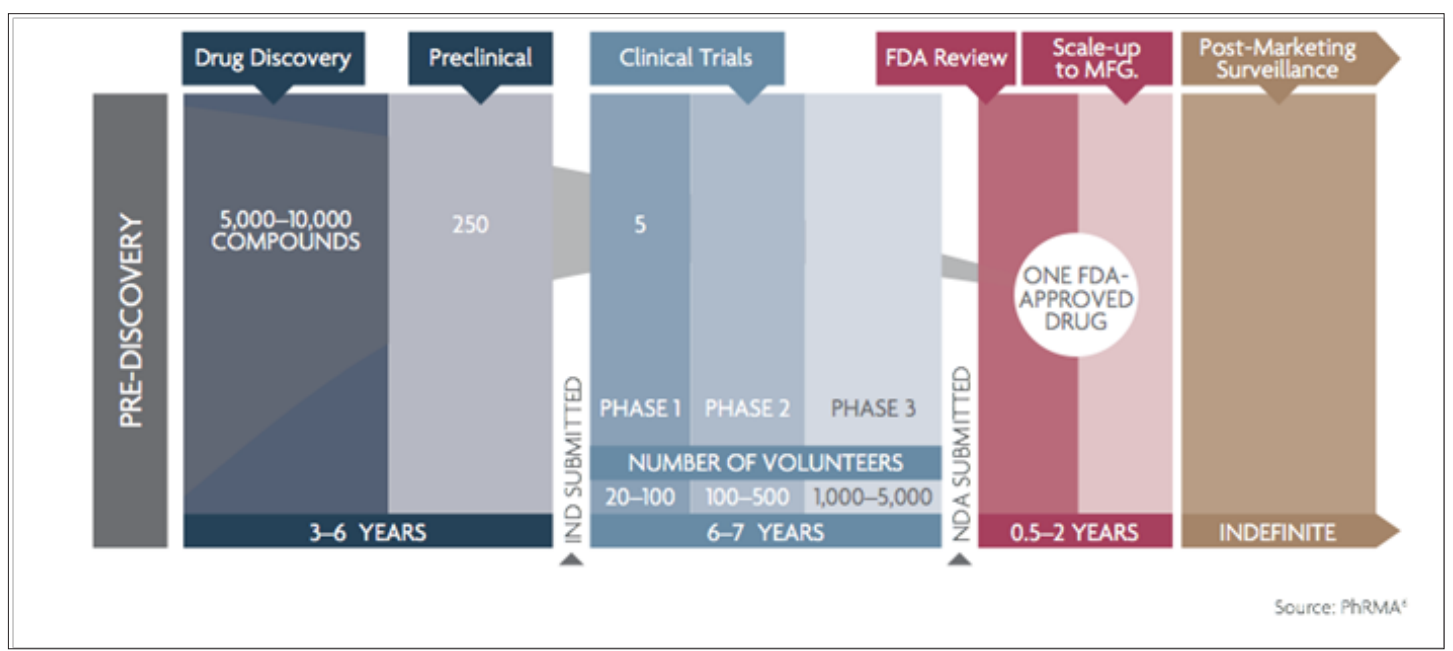

Figure 1: Drug Development Timeline by PhRMA. 
As illustrated in (Figure 1) below and going through different Gantt charts coming from different websites, pharmaceutical development is not a popular topic in the overall drug development scheme and especially for startup companies. Below summarizes somehow most of the drug development pathways as illustrated in pharmaceutical companies and agencies. Two main conclusion scan be extracted from this above timeline: a) Things are presented sequentially but that in reality they are done in parallel.

b) Clinical steps are starting right after the preclinical steps, increasing the statement that PR\&D is not something very popular in drug development overall.

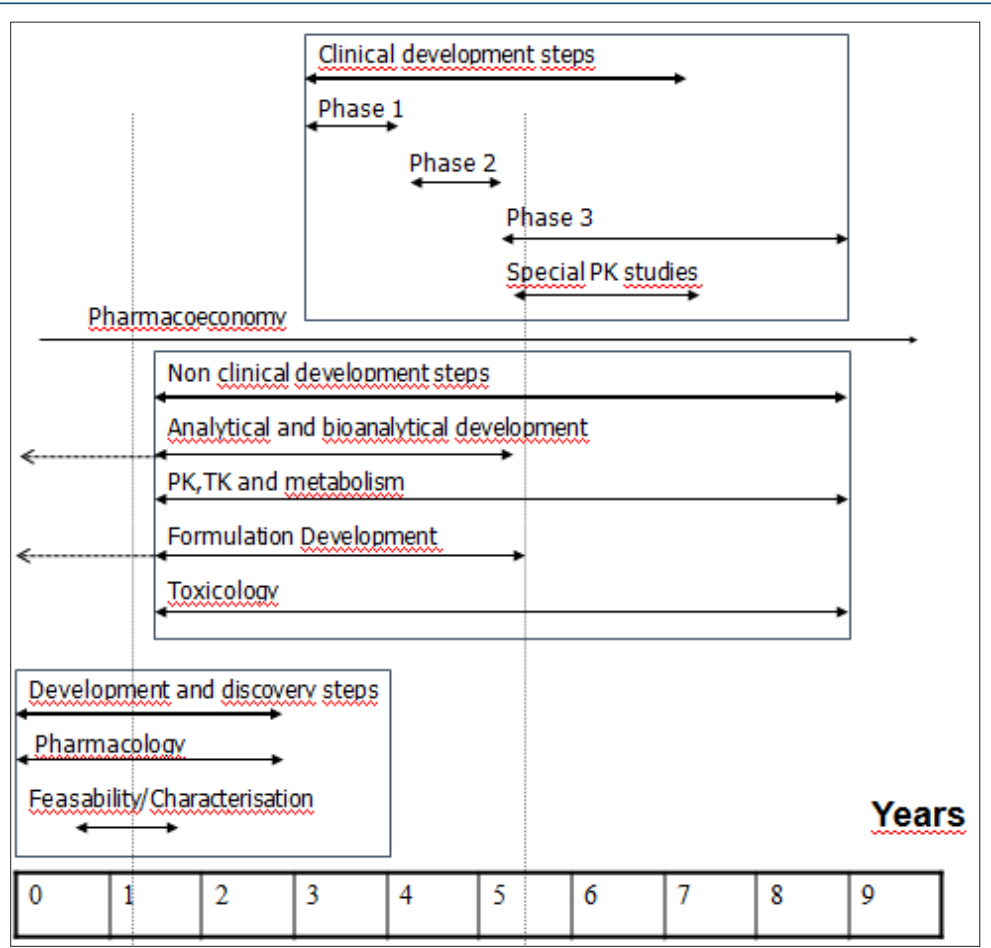

Figure 2: Interaction and timing of the different drug development steps. Adapted and modified from Modern Pharmaceutics ( $4^{\text {th }}$ edition, ed. Banker and Rhodes).

The (Figure 2) above illustrates, in our sense how drug development should be done, regardless of the NME. The following things should be kept in mind then:

a) Many drug development activities are done in parallel (and not sequentially).

b) PR\&D should start almost during drug discovery, or when lead compounds (and backups) have been selected.

From a regulatory standpoint, according to Pharmaceutical Development ICH Q8 Guideline, the aim of pharmaceutical development is to design a quality product and its manufacturing process to consistently deliver the intended performance of the product. It means that irrespective of the development steps and the scale, both the NME and the dosage form should be reliable, reproducible, stable and develop in such a way thatthe changes (scale-up, fine tuning) through development should not require, in a ideal world, bridging studies. A lot of people working in the PR\&D area have noticed that this last ICH Q8 was more than a challenge to achieve. It represents one of the reasons why drug development will hurt. Questions are then the following:

\section{How it can and will hurt- Where does it start?}

a) With the NME: although drug development requires a scientific approach, science should be used to meet regulatory requirements.

b) The NME needs to be developed into an active pharmaceutical ingredient (API) irrespective of its indication.

c) Even though outstanding results were obtained during the proof of concept (POC), a NME is not in itself an API, even less a dosage form.

\section{Why do we not know our API?}

Typical Biotech/start-up situation:

a) Licensed technology/molecule from a university or research center, where the scope and objectives (research is oriented to advance science and knowledge) do not totally meet those of the pharmaceutical industry (research is oriented to serve medical needs within the confines of regulations ultimately allowing bringing a drug product to market). 
b) High knowledge of the chemistry, the biology, the proof of mechanism (not necessary the physiopathology).

c) Lack of knowledge of the pharmaceutical sciences /drug development process.

d) In the quest for nanomolar binding efficiency and biological potency, there has been a gradual shift of new pipeline compounds biopharmaceutical characteristics into less "druggable" compounds. The result then is a higher challenge to develop and maintain a reliable/reproducible dosage form regardless of the scale and the development steps.

\section{Why do we not know our API?}

Typical Biotech/start-up situation

a) Lack of available funds for several reasons (due diligence, overhead, routes of administration were different during nonclinical steps resulting in lack of reliability/efficacy, poor NME characterization at the gram scale, poor development plan).

b) Money is kept for what is perceived as absolutely necessary: the clinic whereas the phase 1 clinical trial may not be the most expensive step (depending the indication), CMC, formulation development and nonclinical could be extremely expensive for a sterile biological product.

c) Need to deliver something quickly.

d) Public companies are driven by market expectations.

e) Private companies need results to get financing.

f) Prepare samples for pre-clinical/toxicological studies with available material.

g) Most of the time, formulations used in preclinical and toxicological studies are not optimized and do not reflect the formulation to be brought to clinical studies.

h) Lack of knowledge of the regulatory requirements for drug development and therefore the associated cost.

i) Drug development is a lengthy process and the regulatory environment changes, so will the cost.

\section{When does it hurt?}

a) Throughout the drug development process.

b) Typical biotech situation: jumping in the drug development arena without required knowledge and expertise, relying on CMOs and CROs to fill the gap.

c) When trying to secure a business partner.

d) Typical biotech situation: licensing after a phase IIA, which needs an un neglect able amount funds. e) Usually a Big Pharma with a high knowledge of drug development process.

f) Due diligence can be deadly: team of experts with high expectations on availability of data, QA audits.

g) When preparing the market application.

h) After commercialization middle size pharma are buying products to fill their pipelines where most of the challenges lie in chemistry, manufacture and controls.

i) Getting out of the laboratory: going to the pilot plant up to GMP facilities.

j) What works at the gram scale usually does not work at the kilo scale.

k) Process parameters change (equipment train, operating conditions, crystallization/purification solvents, mass and heat transfers, etc.)

l) Preparation of the test articles for GLP toxicological studies may not be reliable from the bench to the CRO, root cause being unknown because not investigated.

m) All these impact the quality attributes of the Apian therefore the rest of the drug development chain.

\section{Due Diligence Topics}

NME:

a) Synthesis: yield and scale-up feasibility.

b) Analytical development: wet and solid-state chemistry (which is neglected despite the lack of solubility, the high log $\mathrm{P}$ of the more recent NME).

c) Comments on whether there are any correlations between physical characteristics and formulation orbio availability (polymorphism is somehow neglected).

Non-clinical development and GLP toxicology:

a) How was the proof of mechanism demonstrated (versus gold standard? Route of administration? Reliability?).

b) There is evidence that most sponsors have a good understanding of the toxicology program that is required to bring a product to Phase I; however, the rest of the early phases of drug development (i.e. the development of the actual drug product in a suitable dosage form) is not given the same level of attention despite the increasing poor "drug ability" properties of modern-day NMEs.

c) Current Approaches to Fisrt-In-Human Phase I Clinical Supplies.

d) "Formulated" product approach: Clinical formulation that is a precursor of the desired commercial formulation. 
e) Exploratory formulation approach: Uses the simplest possible formulation (e.g. NME only in bottles or capsules).

f) Keeping in mind that the focus of Phase I testing is mainly to evaluate safety, which approach is applicable and why?

g) Phase I-II clinical programs are rarely rejected from a clinical standpoint. But care should be taken with the clinical supplies: was the formulation the same than during nonclinical proof of concept and GLP toxicological studies? If the formulation has changed, has it modified the Maximum Tolerated Dose, the MEC (minimum effective concentration to get a PD effect), and the No Observed Adverse Event Level (NOAEL)?

\section{When and how does it hurt?}

a) If the NME is not well characterized (frequent), the changes that occur are difficult to identify and the basic quality attributes cannot be properly maintained. It will then generate a snow balling effect throughout drug development.

b) What is the impact of the NME manufacturing (physicochemical characteristics) and formulation development processes (differences in the formulation from non-clinical POC, GLP tox studies, and from phase I to III, scale up) on drug product performance, especially since drug are getting less and less soluble and then "druggable".

c) CMC: preparation of early phase clinical application documents, and modules 2 and 3 of the CTD.

d) Happens after years of drug development initiation.

e) Very often, data was generated way back by people that are no longer in the organization.

f) Characterizing the impurity profile is important not only from a CMC standpoint but also for toxicology and clinical studies. Impurities present at levels above ICH standards should be qualified.

g) If the NME is not well characterized, changes that occurred through the various phases of development cannot be identified and the basic quality attributes cannot be properly maintained.

h) Wet chemical characterization is not enough to portray the complete NME behavior Physical characterization is essential.

i) If there a relationship between the structure and the activity of an NME, (complex NME and biologics), bioassays are more than recommended (and mandatory for most of the agencies).

j) Both the NME and the dosage form will go through "Site changes", going from "laboratory" to "pilot" and then to production scale. k) Site change: bench top scale to cGMP (kilo and pilot scales) facility.

l) cGLP non-clinical, phase I/II clinical studies: safety and "exploratory (dose ranging/finding)" efficacy data acquisition.

m) Impurities? (Safety and CMC are concerned).

n) Polymorphic forms? (CMC, safety and efficacy are concerned) ite changes: from cGMP pilot to cGMP commercial scales.

o) Phase III clinical studies: generation of safety and efficacy data.

p) Pivotal clinical studies.

q) Should use the « final and best » NME and dosage form.

r) Surprises are not welcome at this late stage.

s) When submitting a market application, it is necessary to demonstrate a similarity between the batches used in the clinic and the formula of the proposed commercial product.

t) This applies to both the NME and the dosage form.

u) Late surprises can lead to questioning the validity of clinical batches that have generated data and support the safety and efficacy of the commercial product.

\section{Pre clinical and non-clinical sections}

a) How has the POC been demonstrated (Which lot? Which physico-chemical characteristics? Versus standard of care? Route of administration? Animal model? Reproducibility and reliability? (Ex: IC50 (in vitro)/Cmax (in vivo) if the formulation may impact the way the NME may be absorbed, the Cmax may change form one formulation/experiment to another, the ratio may become biased.

\section{Clinical}

a) Closely connected with the above mentioned poor "druggability", the current approaches for early clinical phases (I/IIa) in drug development

b) Approach with formulated products, where clinical batches will be precursors based on commercial formulation (almost $90 \%$ of the final formulation).

c) Exploratory approach: use the simplest formulation: powder in a bottle or capsule.

d) Keep in mind that the goal of Phase I is safety and pharmacokinetics (even if cohort (s) of patients are part of phase IB).

e) (Figure 3) illustrates the risks of using a non-optimal formulation in phase I/IIa:

f) Increased nonlinear or proportional AUC due to solubility problems (saturation of absorption). 


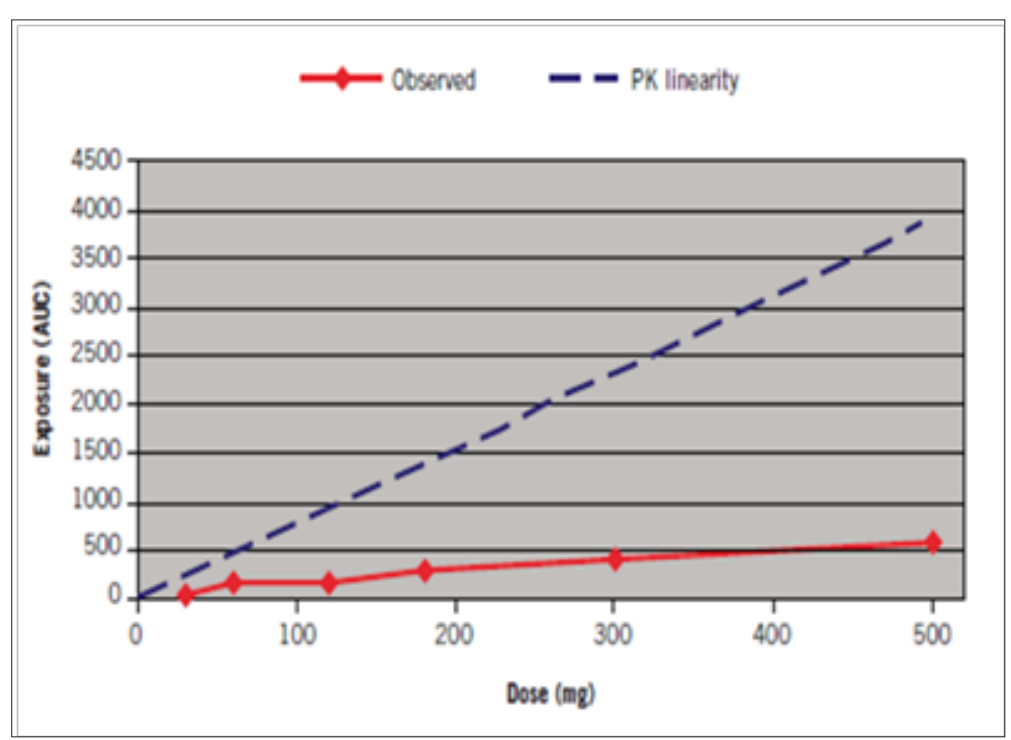

Figure 3: Theoritical and schematic representation of observed AUC and PK linearity vs Dose in mg.

g) No conclusions can be drawn about MTD (maximum tolerated dose, NOAEL, and safety margin,....

\section{What can be done to prevent this?}

a) A good knowledge of the drug development process

b) Get familiar with regulatory requirements at the various stages of development; do not rely only on CROs or consultants...

c) Get the required expertise and build strategies early for pharmaceutical development.

\section{Evolving Regulatory Requirements}

\section{Phase I studies}

a) Brief and focus is on safety, PK and if possible, trend of efficacy (biomarkers, endpoint,..).

b) Clinical design are evolving: SAD/MAD studies, food effects, patients, Therefore the goal of getting an "almost" final formulation is getting more and interesting to increase the reliability form one phase to another. Sponsors do not want the formulation to be held responsible of unexpected results.

\section{Phase II studies}

a) Evidence to reasonably support the proposed chemical structure of the drug substance should be provided: what is the impact on tox, formulation development, clinical studies (dose ranging-finding) and primary efficacy results.

\section{Phase III studies}

a) Final market image! Biobatches, product monograph and expiry date will be based on this scale.
Late surprises may lead to question the validity of clinical batches to support efficacy and safety of the propose commercial product.

\section{Conclusion}

In conclusion, a NME should be developed with complementary people, such as the steps illustrated in (Figure 3) the fact that people get a scientific degree does not make them specialist in all the fields. The regulations will change over time: and so will scientific knowledge and technical/analytical capabilities. It should be kept in mind that Guidance/Guideline documents represent current thinking of regulators and that some are withdrawn and new ones are issued. These documents must be interpreted in the context of the product being developed. There should be a harmonized balance between regulatory and technical/scientific requirements. Knowledge about the NEM should let people streamline their drug product correctly (safety and efficacy) through the whole drug development process, keeping in mind that everything is done in parallel, not sequentially. Since people (from investors to scientists and developers) do not share the same language, it is highly recommended to try to hire the relevant expertise as early as possible, at the early stage, better drug development strategies will be developed planned and well budgeted resulting in a nice risk management position, where "manageable" surprises only may occur down the road, without jeopardizing any launching. 
This work is licensed under Creative Commons Attribution 4.0 License

Submission Link: Submit Article

DOI: $10.32474 /$ DDIPIJ.2018.01.000101

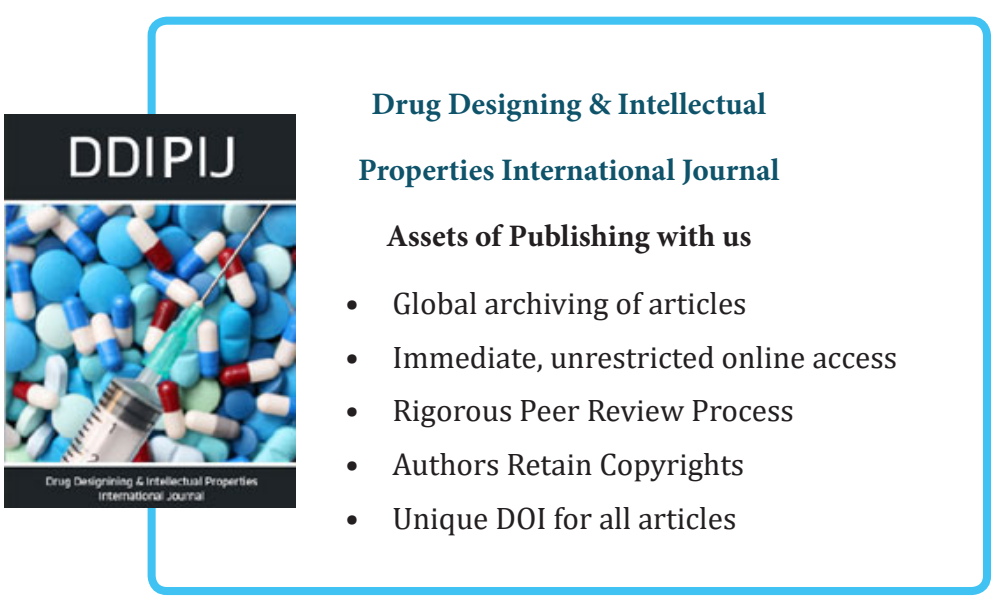

